\title{
Human-scale lung regeneration based on decellularized matrix scaffolds as a biologic platform
}

\author{
Keiji Ohata ${ }^{1,2} \cdot$ Harald C. Ott ${ }^{1,2}$
}

Received: 19 February 2020 / Accepted: 6 March 2020 / Published online: 4 May 2020

(c) The Author(s) 2020

\begin{abstract}
Lung transplantation is currently the only curative treatment for patients with end-stage lung disease; however, donor organ shortage and the need for intense immunosuppression limit its broad clinical application. Bioartificial lungs created by combining native matrix scaffolds with patient-derived cells might overcome these problems. Decellularization involves stripping away cells while leaving behind the extracellular matrix scaffold. Cadaveric lungs are decellularized by detergent perfusion, and histologic examination confirms the absence of cellular components but the preservation of matrix proteins. The resulting lung scaffolds are recellularized in a bioreactor that provides biomimetic conditions, including vascular perfusion and liquid ventilation. Cell seeding, engraftment, and tissue maturation are achieved in whole-organ culture. Bioartificial lungs are transplantable, similarly to donor lungs, because the scaffolds preserve the vascular and airway architecture. In rat and porcine transplantation models, successful anastomoses of the vasculature and the airway were achieved, and gas exchange was evident after reperfusion. However, long-term function has not been achieved because of the immaturity of the vascular bed and distal lung epithelia. The goal of this strategy is to create patient-specific transplantable lungs using induced pluripotent stem cell (iPSC)-derived cells. The repopulation of decellularized scaffolds to create transplantable organs is one of possible future clinical applications of iPSCs.
\end{abstract}

Keywords Lung regeneration $\cdot$ Extracellular matrix $\cdot$ Scaffold $\cdot$ Lung transplantation

\section{Clinical background}

Currently, lung transplantation is the only curative option for patients with end-stage lung disease; however, its broad clinical application is limited by donor organ shortage, the need for immunosuppression, and chronic lung allograft dysfunction [1]. Recently, the discovery of induced pluripotent stem cells (iPSCs) has opened new opportunities in regenerative medicine [2,3], and there has been increasing interest in translating stem cell therapy into clinical application. Despite ongoing progress in the field of regenerative medicine, the development of parenchymal organ engineering has

Harald C. Ott

hott@mgh.harvard.edu

1 Center for Regenerative Medicine, Massachusetts General Hospital, Harvard Medical School, 185 Cambridge Street, CPZN 4800, Boston, MA 02114, USA

2 Division of Thoracic Surgery, Department of Surgery, Massachusetts General Hospital, Harvard Medical School, Boston, MA, USA slowed because of the high levels of architectural and functional complexity of vital organs. In the field of regenerative respiratory medicine, several research groups have reported successful differentiation of human stem cells toward pulmonary epithelial cells of various types, including both distal alveolar type II cells and proximal airway epithelia [4-11]. However, the successful functional engraftment of these cells into injured lungs has not yet been accomplished, and no optimal implantation strategies for the cells have been developed, despite their great therapeutic potential.

Our approach to these hurdles is to use whole-organ matrix scaffolds as a platform to bioengineer a functional organ. We build on the strategies of tissue and organ engineering to combine cells with biocompatible scaffolds to generate biologic devices to replace lost function [12, 13]. This review summarizes our achievements to date and discusses the role of lung matrix scaffolds in lung bioengineering. 


\section{The role of extracellular matrix scaffolds as a biologic platform}

The extracellular matrix (ECM) is a three-dimensional (3-D) molecular network that provides structural and biochemical support for embedded cells. ECM was initially considered simply as a space-filling component of tens of trillions of cells in the human body [14]. We know now that ECM provides essential extracellular environments for multiple types of cells and has a profound impact on cell growth, survival, and differentiation. ECM is first synthesized by embryonic cells during intrauterine development, and cell-ECM interactions are continuous, serving to influence gene expression and determine the cell behavior $[15,16]$. Specifically, the interaction between the ECM and the cellular receptors regulates cell behavior and tissue morphogenesis. Among several important observations, the ECM of muscle was found to promote cell maturation of muscle progenitors as well as differentiate embryonic stem cells to maturing muscle tissue [17]. Likewise, fibronectin, a major component of the ECM, supports the attachment of rat alveolar type II epithelial cells [18]. In wound healing, the ECM promotes the migration and proliferation of progenitor cells and accelerates wound closure [19].

The main components of the ECM include collagens, glycoproteins (such as laminin and fibronectin), proteoglycans, and others, including elastin and hyaluronic acid. Collagen is the major structural component of the ECM and type I collagen is the most abundant subtype detected in tissues. Type IV collagen is a key component of the basement membrane and is among the most important factors to establish and maintain the "air-blood barrier" in the lungs. Laminin and fibronectin are both essential for cell survival. Cells attached to these components receive signals that have immediate impact on their morphology, motility, and differentiation [20, 21]. Proteoglycans are a diverse group of core proteins linked to sulfated polysaccharides or glycosaminoglycans. They bind growth factors and cytokines and retain them within the matrix. Elastin contributes to the mechanical nature of the lung and is a key feature of tissue recoil, which is critical for ventilation. These biologic characteristics are widely applied for in vitro cell culture methods and pharmacotherapeutic fields [22, 23]. As such, a key challenge is to determine how to reproduce the 3-D matrix architecture and promote physiologic ECM-cell interactions and functional gas exchange in lung tissue.

\section{Decellularization of cadaveric lungs}

\section{Strategy for lung decellularization}

In 2008, our group reported the development of decellularized whole-organ scaffolds as platforms for regenerative medicine [13]. Lung decellularization can be accomplished by several techniques, all involving perfusion throughout the native vascular system [24-28]. Decellularization is the process via which all cellular components from cadaveric organs are removed, resulting in isolated natural ECM scaffolds. The end points of adequate decellularization include removing all cellular material and minimizing loss of ECM composition, while at the same time minimizing disruption of the ultrastructure. Different types and combinations of detergents have been successfully employed toward this goal, including sodium dodecyl sulfate (SDS, anionic detergent), sodium deoxycholate (anionic), Triton X-100 (nonionic), Tween (nonionic), and 3-[(3-cholamidopropyl) dimethyl ammonio]-1-propanesulfonate (CHAPS, zwitterionic detergent). Tsuchiya et al. reported that CHAPS solution at low $\mathrm{pH}$ suppressed loss of glycosaminoglycans and elastin, but was not effective at removing all cellular DNA [29]. Dextrose pretreatment might be useful for increasing protein stability and reducing collagen loss during decellularization [30], and additional airway perfusion with detergent solution is an option for more effective decellularization [31]. An attempt to generate vascularized scaffold grafts was performed by a selective cell removal process that targeted only airway cells while keeping vascular cells intact [32]. Importantly, this procedure is applicable to any cadaveric solid organ, including the heart [25, 33], liver [34], kidney [35], pancreas [36], small intestine [37], and limbs [38].

Acellular lung scaffolds were obtained via perfusionbased decellularization of cadaveric lungs. Detergent perfusion effectively removed cellular material from the tissue by solubilizing cell membranes. Table 1 and Fig. 1 include our decellularization protocols and systems used for rat lungs (Fig. 1a) and also for larger lungs from swine, monkeys, and humans (Fig. 1b). Lungs procured from heparinized donors were sequentially perfused with SDS solution, distilled water, Triton X-100 solution, and phosphate-buffered saline through a cannula placed in the pulmonary artery within custom-made decellularization chambers. The perfusion with the SDS solution removed cellular components and the $1 \%$ Triton X-100 wash step facilitated the removal of residual SDS within the tissue. Vascular resistance increased abruptly on the initiation of SDS perfusion because of the high viscosity of the solubilized cellular content, and gradually decreased as the cellular content decreased; as such, constant and controlled low-pressure perfusion provided optimal results. We maintained a perfusion pressure below $15 \mathrm{mmHg}$ during the decellularization process to prevent breakage of the very friable capillary basement membranes. Decellularization of human and large animal lungs required higher concentrations of detergent solution and longer perfusion times than those used in rodents. We found that the lungs lost opacity as the cellular material was washed out (Fig. 2a). DNA and protein content of the decellularization 
Table 1 Decellularization protocols for rat lungs and larger lungs from swine, monkeys, and humans

\begin{tabular}{|c|c|c|c|c|}
\hline \multicolumn{5}{|c|}{ Decellularization protocol for rat lungs } \\
\hline & Solution & Concentration & Perfusion flow & Duration \\
\hline 1 & SDS & $0.1 \%(\mathrm{wt} / \mathrm{vol})$ & Gravity pressure of $80 \mathrm{mmHg}$ & $120 \mathrm{~min}$ \\
\hline 2 & Distilled water & & Gravity pressure of $80 \mathrm{mmHg}$ & $15 \min$ \\
\hline 3 & Triton X-100 & $1 \%(\mathrm{vol} / \mathrm{vol})$ & Gravity pressure of $80 \mathrm{mmHg}$ & $10 \min$ \\
\hline 4 & PBS & & Roller pump flow of $4 \mathrm{ml} / \mathrm{min}$ & $2-3$ days \\
\hline \multicolumn{5}{|c|}{ Decellularization protocol for lungs from swine, monkeys, and humans } \\
\hline & Solution & Concentration & Perfusion flow & Duration \\
\hline 1 & SDS & $0.5 \%(\mathrm{wt} / \mathrm{vol})$ & Constant pressure of $10-15 \mathrm{mmHg}$ with roller pump & $30 \mathrm{~h}$ \\
\hline 2 & Distilled water & & Constant pressure of $10-15 \mathrm{mmHg}$ with roller pump & $12 \mathrm{~h}$ \\
\hline 3 & Triton X-100 & $1 \%(\mathrm{vol} / \mathrm{vol})$ & Constant pressure of $10-15 \mathrm{mmHg}$ with roller pump & $12 \mathrm{~h}$ \\
\hline 4 & PBS & & Constant pressure of $0-15 \mathrm{mmHg}$ with roller pump & $2-3$ days \\
\hline
\end{tabular}

$S D S$ sodium dodecyl sulfate, $P B S$ phosphate buffered saline a

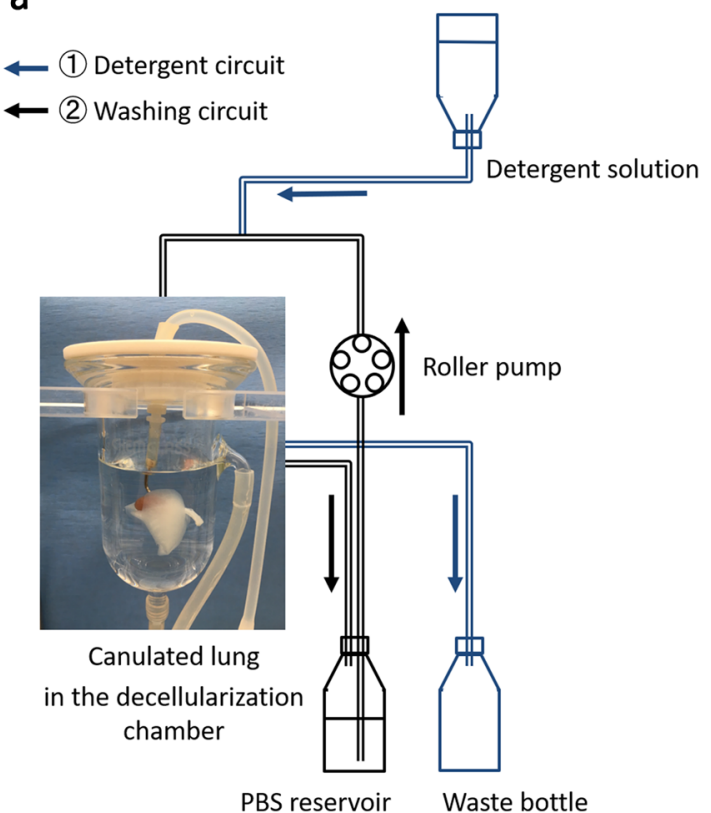

Fig. 1 The decellularization systems for rat lungs (a) and larger lungs from swine, monkeys, and humans (b). a Lungs procured from heparinized donors are sequentially perfused with sodium dodecyl sulfate (SDS) solution, distilled water, and Triton X-100 through a cannula placed in the pulmonary artery under a gravitational pressure of $80 \mathrm{mmHg}$. Detergent solution is infused into the lung through pulmonary arterial canula under a gravitational pressure of $80 \mathrm{mmHg}$ and

effluent also decreases gradually in association with the change in lung appearance (Fig. 2b) [39]. We also successfully scaled up the decellularization process to be achieved for lungs from pigs, monkeys, and humans (Fig. 3). b

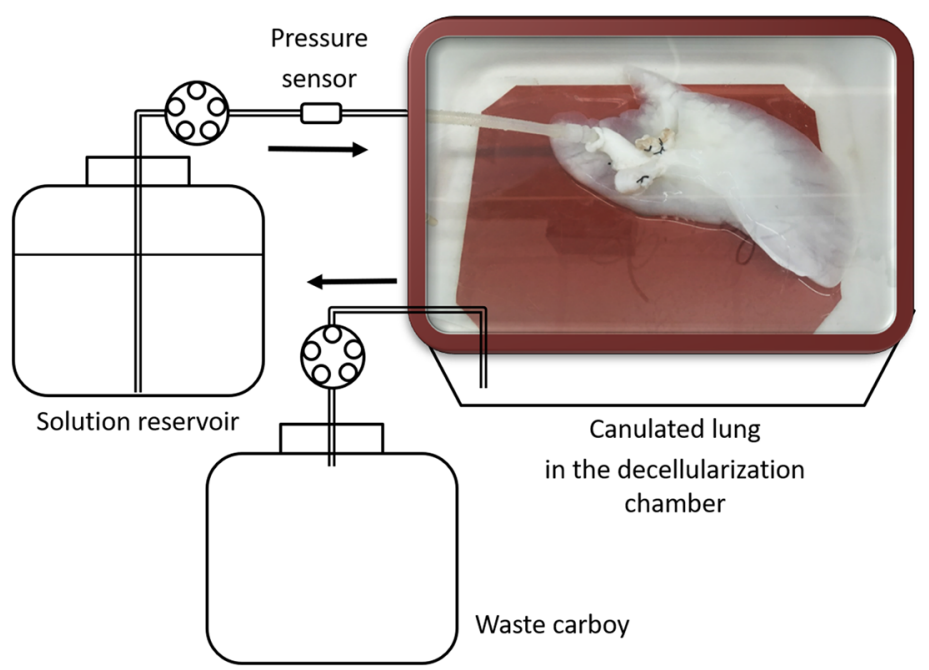

the effluent is drained to the waste bottle (blue arrow). After detergent infusion, phosphate buffered saline (PBS) circulates the circuit by the roller pump and wash the lung (black arrow). b The porcine left lung is placed in the custom-made decellularization chamber and perfused with detergent solutions and PBS through the pulmonary artery. The flow rate is controlled by the roller pump to maintain a perfusion pressure below $15 \mathrm{mmHg}$. The effluent is drained to the waste carboy

\section{Key features of decellularized lung scaffolds as a biologic platform for lung regeneration}

Decellularized native scaffolds preserve both the microand macroarchitecture of the native lung and retain much of the original ECM composition. One of the difficulties inherent in creating any parenchymal organ is the necessary 
a
Cadaveric lung
$6 \mathrm{hr}$
$18 \mathrm{hr}$
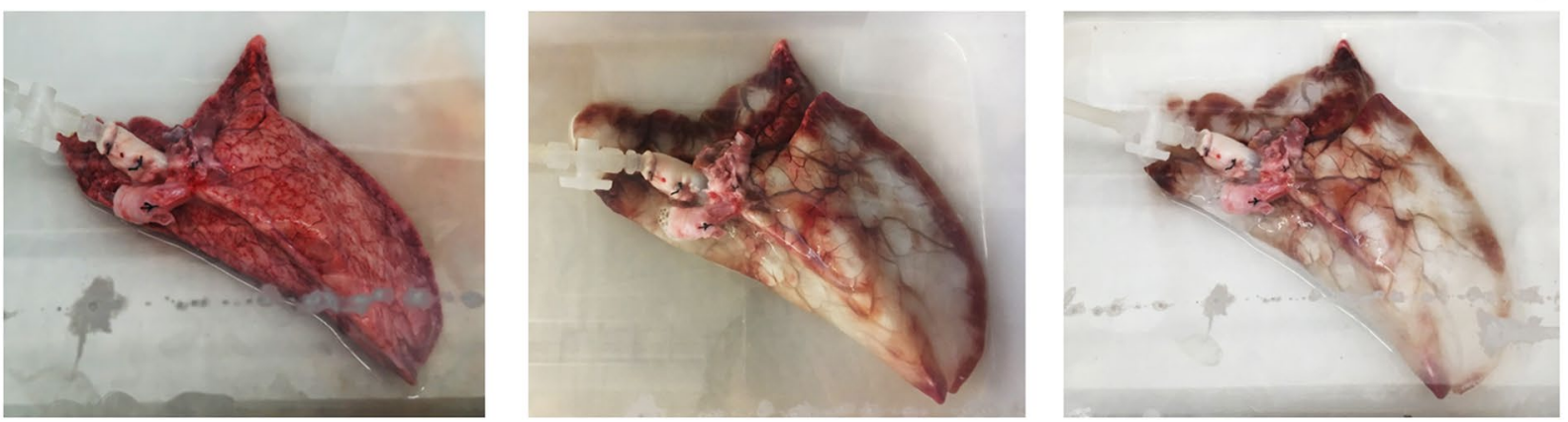

$24 \mathrm{hr}$

After decellularization

b
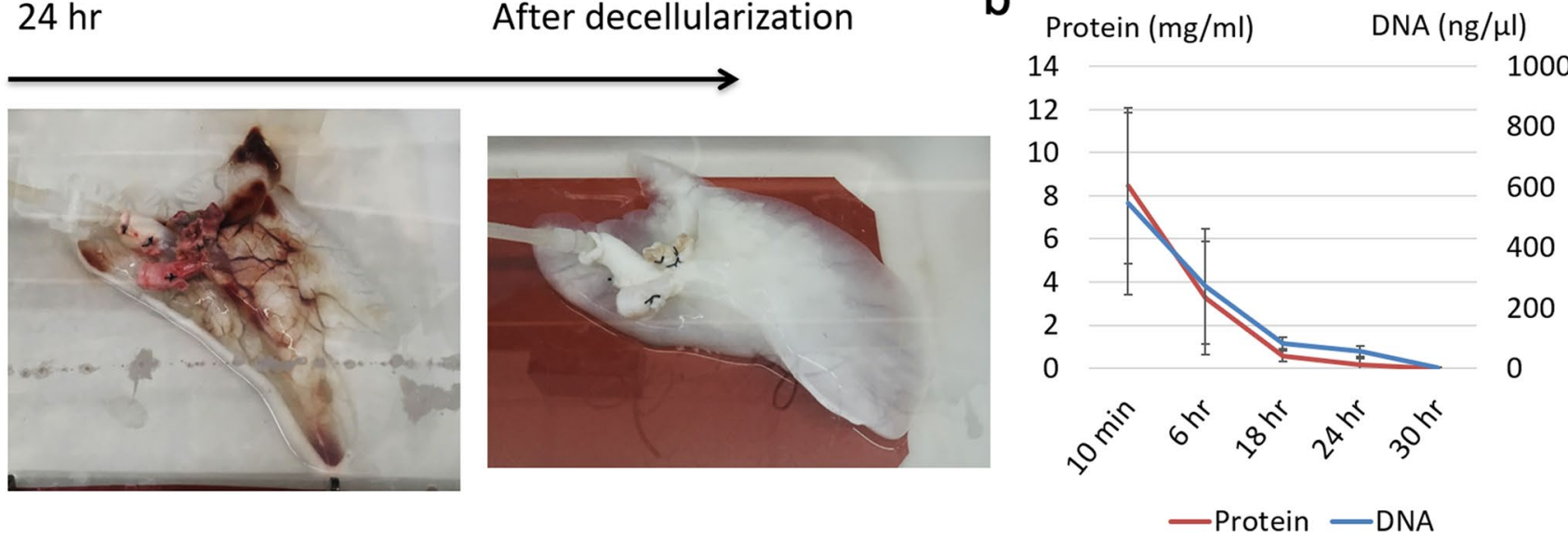

Fig. 2 a The gross appearance of changes in the porcine lung during decellularization process. The lung lost opacity as the cellular material was washed out. b DNA and protein content in the effluent gradually decreased in association with the change in lung appearance

reproduction of its critical and complex 3-D architecture. The minimum functional unit of the lung is the highly specialized "air-blood barrier" between the alveoli and capillaries. Gas exchange requires close interaction between interalveolar air and blood circulation across the alveolar epithelium, basement membrane, and vascular endothelium. Reproducing this ultrathin barrier using conventional manufacturing techniques has not been accomplished to date. Decellularized lung scaffolds preserve the 3-D microarchitecture of the lungs and enable the alveolar epithelium and vascular endothelium to form monolayers with a basement membrane and intact barrier function and basement (Fig. 4) [39]. Furthermore, alveoli and capillaries are connected within hierarchical conducting systems, the bronchial tree, and the vascular network, respectively, which facilitates near-immediate contact between ambient air and circulating blood. Preservation of the intact vascular network and bronchial tree allows direct connection between the transplant and the vasculature and airway, either orthotopically or heterotopically. The hierarchical branching system also enlarges the alveolar surface area and maximizes the efficiency of gas exchange. Even with advanced manufacturing technology, including 3-D printing, the complex architecture of the lung has not been reproduced successfully; thus, acellular lung scaffolds remain the most promising approach.

Decellularized native scaffolds retain some of the original ECM composition and can provide cells with site-specific support. Histologic analysis of a porcine lung acellular scaffold showed no cellular nuclei, but preservation of type I collagen, laminin, and fibronectin in the decellularized lung (Fig. 4) [39], thereby providing the unique and critical organ-specific environment for cell growth and maturation. Some ECM components are inevitably lost in any decellularization protocol, and quantitative measurements of ECM components remaining after perfusion have revealed reductions in elastin and sulfated glycosaminoglycans [29, 39]. As an intricately interwoven network of collagen and elastin fibers provides important mechanical support for respiration, the decrease in elastin may contribute to extensive problems with compliance and lack of recoil in the decellularized 
a

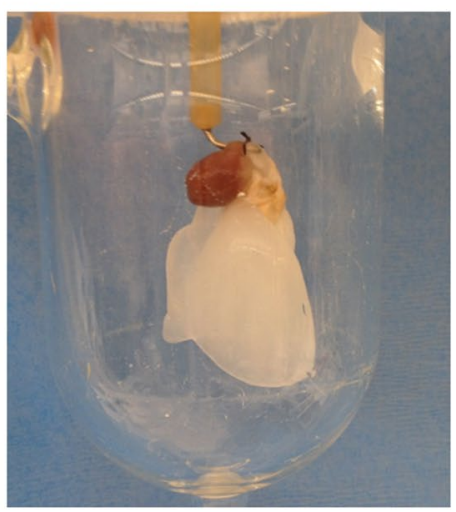

C

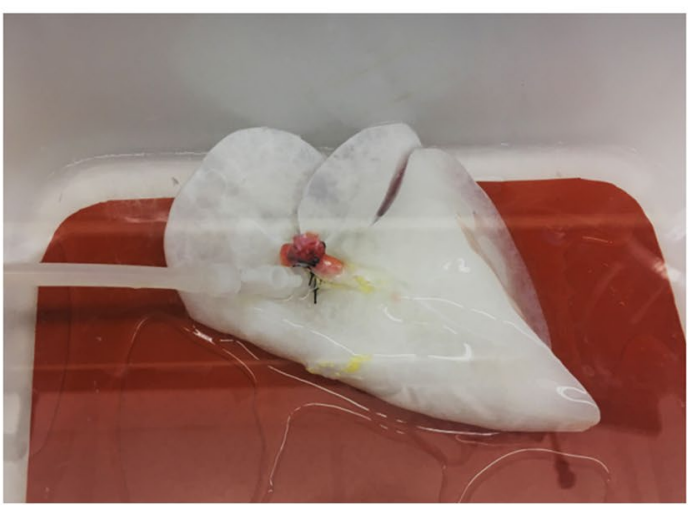

b

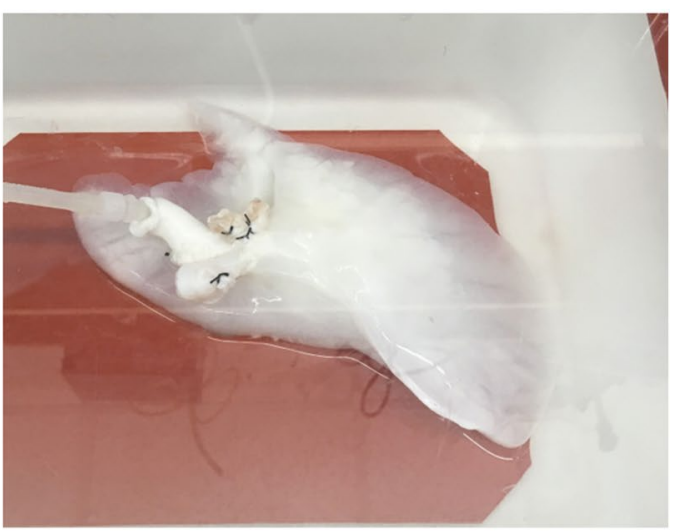

d

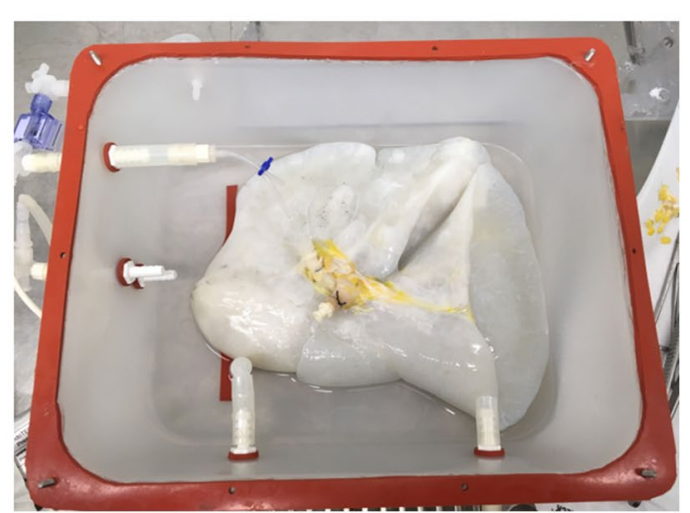

Fig. 3 Gross appearance of decellularized lungs procured from a rat, $\mathbf{b}$ swine, $\mathbf{c}$ macaque, and $\mathbf{d}$ human

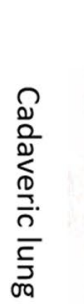

$\mathrm{HE}$

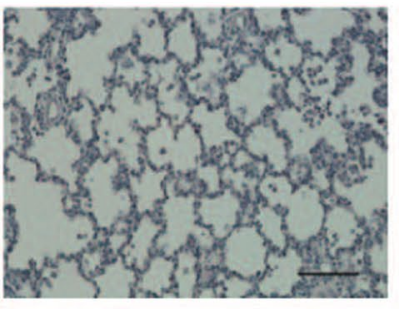

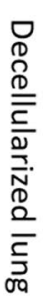

Collagen I
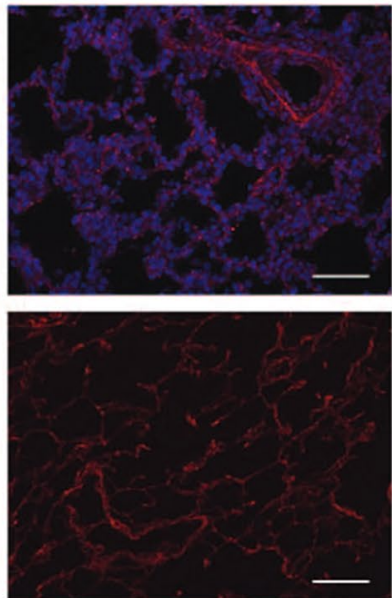

Laminin
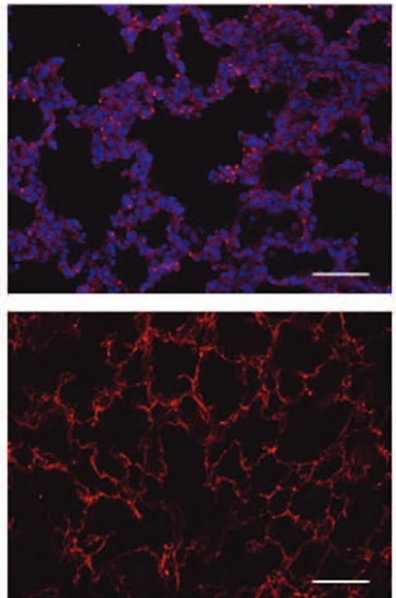

Fibronectin
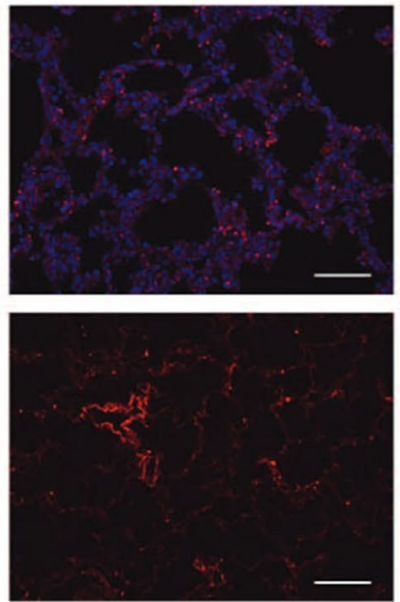

Fig. 4 Hematoxylin and eosin (HE) staining and immunofluorescent (IF) staining of the porcine cadaveric lung and decellularized lung scaffold. HE staining showed that decellularized scaffolds preserve the alveolar structure of the lungs. IF staining revealed the complete absence of cellular nuclei but the preservation of and matrix proteins including type I collagen, laminin, and fibronectin in the decellularized lung. Scale bars $100 \mu \mathrm{m}$ 
lungs. To obtain a more suitable platform for tissue engineering, methods for enhancement of acellular scaffolds are in development. In the cardiovascular field, autologous pericardium treated with a glutaraldehyde solution has been used for aortic valve reconstruction [40]. Glutaraldehyde strengthens ECM by cross-linking collagen, which might be useful for generating whole-organ matrix scaffolds. From a functional aspect, Chen et al. reported that using a decellularized bone scaffold coated with stromal cell-derived factor- $1 \alpha$ improved endogenous stem cell recruitment and enhanced osteogenesis in a rabbit model [41]. We analyzed compositional differences between human neonatal and adult lung ECM and found that fibrillin-2 and tenascin-C were both expressed at higher levels in the neonatal ECM. Accordingly, supplementation with fibrillin-2 and tenascin$\mathrm{C}$ resulted in increased epithelial cell proliferation in both in vitro and ex vivo 3-D scaffold cultures [42].

\section{Xeno-organs as scaffolds for human tissue}

Declined human donor lungs are the most logical and immediate candidates for decellularization and clinical application, as these have already been used extensively for experimental purposes [43, 44]. However, donor lungs are an inherently heterogeneous source and have experienced varying degrees of injury from the circumstances of death and donation. For example, re-seeded cells did not survive beyond 1 week in decellularized emphysematous human lungs; however, this scaffold may lack the essential 3-D ECM architecture to support cell growth [45]. Likewise, matrix scaffolds created from the lungs of patients with idiopathic pulmonary fibrosis displayed significant compositional differences from the normal lung, and the fibrotic matrices promoted myofibroblast differentiation [45]. The relationship between lung damage and matrix functionality needs to be further investigated.

Scaffolds generated from pig lungs might be a feasible alternative because of the striking anatomic similarities between porcine and human organs. An animal source for these scaffolds would facilitate precise quality control. Porcine scaffolds could be a by-product of the food industry, whereby we could take advantage of a nearly unlimited and highly consistent supply of donor tissue. ECM components are highly conserved across species, and xenogeneic scaffold devices have already been used in clinical practice for a variety of reconstructive procedures, including heart valve replacement and wound care [46]. A major factor underlying the hyperacute rejection of pig organ transplants in primates is an acute reaction to the galactose- $\alpha$-1,3-galactose (Gal)-epitope. However, the Gal-epitope in porcine ECM scaffolds did not elicit a host response or tissue remodeling because of the relatively small amount of antigen present in the tissue [47]. Furthermore, genetically modified $\alpha 1,3-$ galactosyltransferase gene-deleted pigs are now available. The absence of the Gal-epitope from porcine lung tissue delayed immune cell infiltration and reduced the chronic $\mathrm{T}$ cell-mediated reaction against decellularized materials following reimplantation [48]. As such, removing the Galepitope may be an effective strategy for extending the life of clinical-grade decellularized scaffolds. Porcine organs might be an option for whole-organ scaffolds in future clinical application.

\section{Recellularization of lung matrix scaffolds}

\section{Cell types for recellularization and cell infusion strategy}

Optimization of recellularization and maturation protocols is a current challenge in bioengineering clinically useful acellular scaffolds. Repopulation of organ scaffolds requires appropriate cell population, which will provide high-level organ-specific function. The first step in organ scaffold recellularization is a cell infusion procedure in which appropriate cell types are placed at relevant sites in the scaffolds. Techniques to effectively deliver specific cell populations into the correct niches throughout the lung scaffold are being developed.

The biggest challenge in creating bioartificial lungs from scaffolds is the need to reproduce functional and stable vascular networks that play an essential role in the gas exchange function, nutrient supply, and waste transport from the lungs: vascular endothelial cells are the primary population contributing to these functions. For vascular cell re-seeding, cell suspensions are infused simultaneously through the pulmonary artery and pulmonary vein under a gravitational pressure of $80 \mathrm{mmHg}$. Gravity-driven cell infusion contributed to the even distribution of cells within the vasculature and supported cell survival. In contrast, seeding driven by a roller pump device led to increased cell death [49]. The use of low-concentration and high-volume cell suspensions resulted in an even distribution of cells throughout the lung scaffolds. After cell infusion, a static period, followed by simultaneous arterial and venous perfusion, facilitates cell attachment to the matrix. Incomplete endothelial cell coverage within the vasculature promotes thrombosis secondary to platelet activation and adhesion to the ECM or alveolar hemorrhage. Exposed ECM proteins in the scaffold may also invoke aberrant immune responses upon implantation. Collagen type $\mathrm{V}$ has been identified as a critical factor contributing to chronic lung allograft dysfunction [50]. A variety of cells have been used to repopulate the pulmonary vasculature, including primary cells from rat lung 
microvessels [24], human umbilical vein endothelial cells [39], and iPSC-derived vascular endothelial cells [44]. A number of cells that are sufficiently able to obtain cell-cell interactions and paracrine activities within the scaffold should be included in the infusions.

The parenchymal cells responsible for the specific functions of the organ are also necessary. To date, different airway epithelial cells, including a heterogeneous mixture of fetal or neonatal rat lung cells [24, 25], carcinomatous human alveolar epithelial cells [25], human airway basal stem cells [39, 51], and iPSC-derived epithelial cells [7, $52,53]$, have been used to re-epithelialize the airways of lung scaffolds. In an airway re-seeding procedure, the epithelial cell suspension is infused via a bronchus or the trachea. The dead-ended architecture of the bronchial tree makes it difficult to achieve universal and even delivery to all sites. Some approaches that have already been used for intratracheal cell transport and delivery might be adopted for acellular scaffolds [54]. An improved understanding of intratracheal cell transport dynamics and development of suitable cell monitoring techniques are both needed.
In addition to the cells that reconstitute the epithelium and endothelium, other cell lineages need to be incorporated, including pericytes and smooth muscle cells [44, 55]. Likewise, fibroblasts enhance the functional phenotype of the parenchymal cells and contribute to the organization of the tissue architecture. The introduction of undifferentiated lung stem cells into these microenvironmental niches might contribute to tissue homeostasis after implantation for long-term success.

\section{Development of bioreactor and ex vivo organ culture}

The lung scaffolds are recellularized in a bioreactor that facilitates cell delivery and perfusion with culture medium via the pulmonary artery, vein, and airway. After the cell attachment, the cells on the ECM scaffold are maintained in an organ-specific biomimetic environment. Developing specialized bioreactors that support cell growth and maturation within the bioartificial lungs is another important challenge in this field. Some researchers have designed bioreactors a

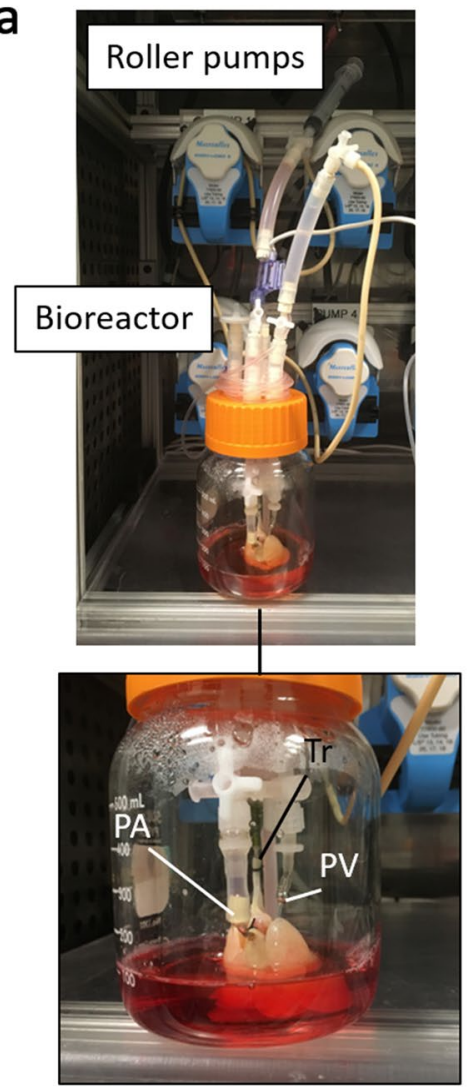

Fig. 5 Our recellularization systems for rat- $\mathbf{a}$ and human-sized $\mathbf{b}$ lungs. Cell seeding, engraftment, and tissue maturation are achieved in the bioreactor, which provides biomimetic conditions, including vascular perfusion and liquid ventilation by roller pumps. An air- b

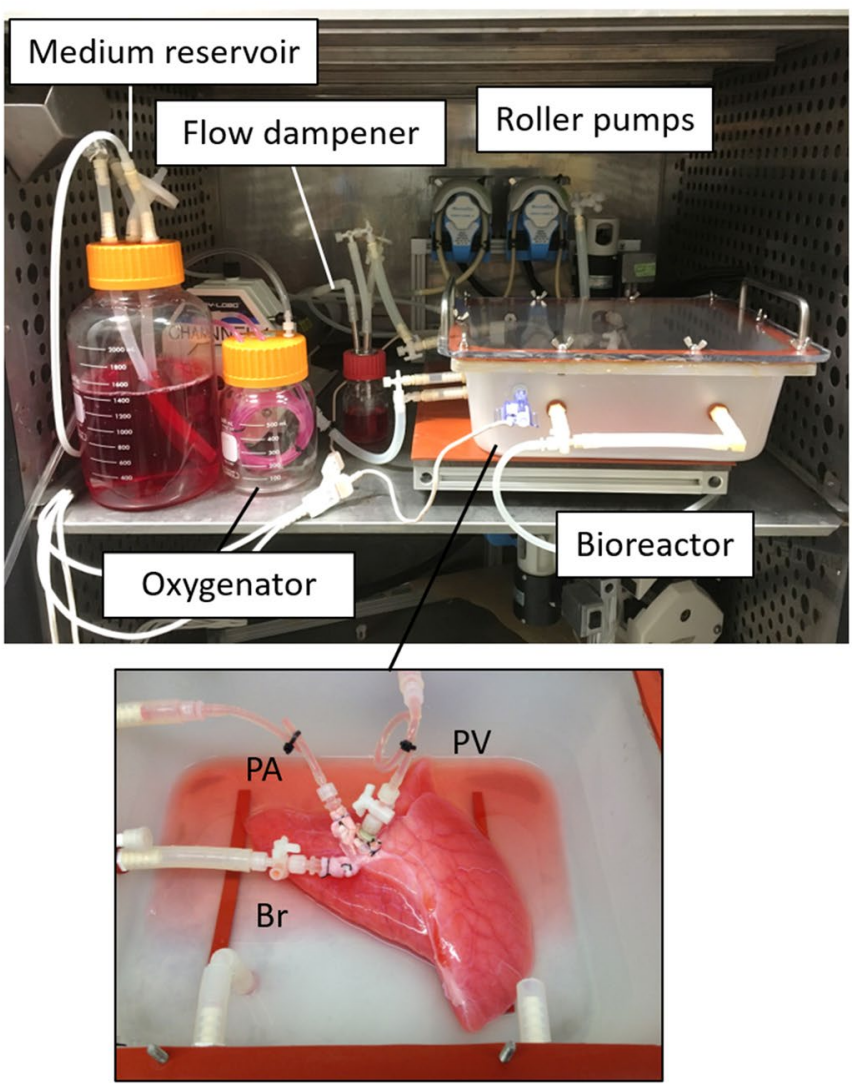

cushioned flow dampener facilitates smooth vascular perfusion that minimizes matrix damage. $P A$ pulmonary artery, $P V$ pulmonary vein, $\operatorname{Tr}$ trachea, $B r$ left bronchus 
that promote nutrient, waste, gas, and cytokine exchange $[56,57]$.

Figure 5 shows our recellularization systems for rat- and human-sized lungs. Whole-organ culture systems are based on extracorporeal organ perfusion devices that drive culture medium through the graft. The lung bioreactor circulates perfusate from a reservoir through the lungs via the pulmonary artery. Perfusion culture with a sufficient flow rate and pulmonary arterial pressure can result in full recellularization of the lung vasculature and promote the delivery of oxygen and nutrition due to the relatively low vascular resistance, a notably crucial feature particularly in human-sized lung scaffolds. Smooth anterograde perfusion that minimizes matrix damage is facilitated by a roller pump with an aircushioned flow dampener. Mechanical stimulation is a key factor that enhances cell growth and organ maturation in organ culture. The flow of culture medium through vascular scaffolds in the bioreactor produces shear stress as a result of hemodynamic forces. This biophysical stimulus promotes homing, paracrine effects, and differentiation of endothelial progenitor cells. An additional oscillation flow might be an option to enhance these beneficial effects. In more recent recellularization strategies, we employed open vein circulation, which includes a venous pressure of $0 \mathrm{mmHg}$ against the hilum. Controlled positive venous pressure may contribute to more effective lung recellularization. In a study of ex vivo lung perfusion with pig cadaveric lungs, a left atrial pressure of $5 \mathrm{mmHg}$ led to reduced edema and superior lung physiology [58].

Airway perfusion is another option in lung organ culture. The movement of medium in and out of the airways is driven by negative pressure in the bioreactor chamber and the addition of cyclic stretch may provide crucial developmental signals [59]. Petersen et al. reported that liquid ventilation promoted cell survival and maintained airway structure in cadaveric rat lung organ culture [60]. Alveolar dilation recruits capillary vessels and may also have a positive impact on the recellularization of microcapillaries. Previous lung organ culture successfully maintained recellularized scaffolds for several days and up to as long as 1 month $[30,39]$. Noninvasive developmental strategies for real-time monitoring of recellularization are needed [61].

\section{Induced pluripotent stem cells as a source for lung recellularization}

As we move toward the most clinically relevant model, ideal cell sources for recellularization must be identified and tested. This will most likely involve iPSC-derived cells. Yamanaka et al. successfully reprogrammed mouse and human fibroblasts to generate pluripotent stem cells $[2,3]$. Repopulating scaffolds with donor-derived cells differentiated from iPSCs would facilitate personalized transplantation medicine and reduce the need for immunosuppressive therapy. The proliferating capacity of iPSCs suggests that it will not be difficult to obtain appropriately differentiated cells in sufficient numbers to regenerate organ function. Autologous primary cells isolated from harvested lung tissue are insufficiently proliferative and do not typically expand to the numbers required for rebuilding on our scaffolds; as such, they are less likely candidates for clinical application. In addition, lung cell collection with the intent to repopulate is not an option for patients with end-stage lung disease. The stem cell-specific microenvironments, also known as "niches" $[62,63]$, which serve to maintain stem cell renewal and differentiation have been described in a number of different organs. ECM proteins are among the most important components of this niche.

Cells would ideally be derived from nonimmunogenic sources, but allogenic cells might serve as candidates. Kyoto University currently maintains recorded stocks of iPSCs to be used for regenerative medicine and research. These stocks include iPSCs induced from healthy donors with defined human leukocyte antigen (HLA) profiles. Xu et al. reported on the use of CRISPR-Cas9-based HLA editing to create the immunocompatible iPSCs [64]. This will undoubtedly become a powerful source of iPSCs for both research and for clinical practice.

\section{Transplantation of bioartificial lungs}

The primary outcome of lung bioengineering studies is in vivo gas exchange. As the lung scaffolds preserve the vascular and airway architecture from alveoli to the hilum, the bioartificial lungs should be transplantable in a fashion similar to that for cadaveric donor lungs. Initially, researchers from Yale University and our group demonstrated the feasibility of recellularized bioartificial lungs in a rat transplantation model [24, 25]. Petersen et al. repopulated acellular rat lung scaffolds with mixed populations of neonatal rat lung epithelial cells and microvascular endothelial cells [60]. We recellularized our scaffolds with carcinomatous human alveolar epithelial cells or rat fetal lung cells combined with endothelial cells. In both studies, the recellularized lungs were transplanted orthotopically into recipient rats, which resulted in partial restoration of respiratory function. After optimization, we created bioartificial rat lungs that were recellularized with rat fetal pneumocytes and human primary vascular cells. These grafts maintained respiratory function through the recipient's airway for up to 7 days after implantation [65].

Scaling up from a small animal to a clinically relevant large animal model is a critical step toward human clinical application. Toward this end, we created human-sized bioartificial lung grafts by repopulating porcine lung scaffolds 
with human umbilical vascular endothelial cells and human basal airway stem cells. These grafts were transplanted heterotopically with the pulmonary artery and vein anastomosed with the pulmonary trunk and left atrial appendage, respectively. The graft withstood physiologic pulmonary blood flow and performed gas exchange for $1 \mathrm{~h}$ after surgical implantation [39]. We also created bioartificial lungs and performed orthotopic transplantation to assess feasibility and in vivo function using a porcine 24-h survival model. Technically successful orthotopic anastomoses of the vasculature and the airway were achieved, and perfusion and ventilation of the lung grafts were confirmed. Gas exchange was evident immediately after transplantation. Unfortunately, the graft lacked sufficient barrier function and an increase in thrombus formation led to graft failure within $24 \mathrm{~h}$. Further progress toward optimizing recellularization and maturation of the grafts will be necessary to improve their sustainability and functionality. Nichols et al. recellularized porcine lung scaffolds with autologous cells from porcine recipients and transplanted them without vascular anastomoses [30]. In this study, the bioartificial lungs survived in the recipient thorax accompanied by further cell expansion and vascular tissue development after implantation.

\section{Application of organ scaffolds to other fields}

Decellularized organ scaffolds also hold promise as a tool for modeling human disease. Lung scaffolds have been used to culture lung cancer cells in a biomimetic 3-D environment, presenting unique aspects of the biology of this disease [66, 67]. Mishra et al. reported that cancer cells in the scaffold produced matrix metalloproteinases that were known products of the cells in situ but were not detected in 2-D culture [68]. Lung scaffolds will also enable us to monitor other cancer cell characteristics under biomimetic conditions including proliferation and the relative sensitivity to chemotherapeutic agents [69].

The contributions of acellular scaffolds to decisions relating to the cell fate of iPSCs are important but incompletely understood. For example, the attachment of embryonic stem cell-derived lung epithelial cells to ECM proteins resulted in enhanced surfactant protein-C expression [70]. Human iPSC-derived lung organoids improved the cellular differentiation, and the resulting airway-like structures were similar to the human adult lung [63]. Modeling of human lung development, lung disease, and screening for effective drug therapies might all be improved with the future use of iPSC-derived cells for this purpose.

\section{Conclusions}

Acellular lung scaffolds from small and large animals, which have preserved the original vascular network, bronchial tree, and most of the ECM composition, were generated via perfusion of the pulmonary artery with detergent solutions. The resulting lung scaffolds were recellularized in bioreactors and successful cell growth was achieved with lung perfusion culture. The resulting bioartificial lungs with the vascular and airway architectures preserved would be used in transplant studies. In rat and porcine transplantation models, the bioartificial lungs that were transplanted promoted gas exchange after implantation, although graft failure resulted from insufficient vascular barrier function and increased thrombogenicity. As such, further progress in optimizing the recellularization and the maturation of the grafts will be necessary to improve the sustainability and the functionality of the grafts. While early in its development, organ engineering provides the unique potential to promote personalized treatment options based on recellularization with patientderived iPSCs.

Funding Several studies quoted in this report were supported by the United Therapeutics Corporation, an NIH New Innovator Award (DP2 OD008749-01 HCO), the NHLBI (R21 HL108663 HCO, R01 HL108678 DK, R01 HL111574-01 LO), the Mendez Foundation, and the Charles and Sara Fabrikant MGH Research Scholarship.

\section{Compliance with ethical standards}

Conflict of interest H.C.O. is a founder and stockholder of IVIVA Medical Inc. This relationship did not affect the content of conclusions contained in this manuscript.

Open Access This article is licensed under a Creative Commons Attribution 4.0 International License, which permits use, sharing, adaptation, distribution and reproduction in any medium or format, as long as you give appropriate credit to the original author(s) and the source, provide a link to the Creative Commons licence, and indicate if changes were made. The images or other third party material in this article are included in the article's Creative Commons licence, unless indicated otherwise in a credit line to the material. If material is not included in the article's Creative Commons licence and your intended use is not permitted by statutory regulation or exceeds the permitted use, you will need to obtain permission directly from the copyright holder. To view a copy of this licence, visit http://creativecommons.org/licenses/by/4.0/.

\section{References}

1. Chambers DC, Cherikh WS, Goldfarb SB, Hayes D, Kucheryavaya AY, Toll AE, et al. The International Thoracic Organ Transplant Registry of the International Society for Heart and Lung Transplantation: thirty-fifth adult lung and heart-lung transplant report-2018; focus theme: multiorgan transplantation. $\mathbf{J}$ Heart Lung Transplant. 2018;37:1169-83. 
2. Takahashi K, Yamanaka S. Induction of pluripotent stem cells from mouse embryonic and adult fibroblast cultures by defined factors. Cell. 2006;126:663-76.

3. Takahashi K, Tanabe K, Ohnuki M, Narita M, Ichisaka T, Tomoda $\mathrm{K}$, et al. Induction of pluripotent stem cells from adult human fibroblasts by defined factors. Cell. 2007;131:861-72.

4. Green MD, Chen A, Nostro MC, D'Souza SL, Schaniel C, Lemischka IR, et al. Generation of anterior foregut endoderm from human embryonic and induced pluripotent stem cells. Nat Biotechnol. 2011;29:267-73.

5. Wong AP, Bear CE, Chin S, Pasceri P, Thompson TO, Huan LJ, et al. Directed differentiation of human pluripotent stem cells into mature airway epithelia expressing functional CFTR protein. Nat Biotechnol. 2012;30:876-82.

6. Mou H, Zhao R, Sherwood R, Ahfeldt T, Lapey A, Wain J, et al. Generation of multipotent lung and airway progenitors from mouse ESCs and patient-specific cystic fibrosis iPSCs. Cell Stem Cell. 2012;10:385-97.

7. Ghaedi M, Calle EA, Mendez JJ, Gard AL, Balestrini J, Booth A, et al. Human iPS cell-derived alveolar epithelium repopulates lung extracellular matrix. J Clin Invest. 2013;123:4950-62.

8. Gotoh S, Ito I, Nagasaki T, Yamamoto Y, Konishi S, Korogi Y, et al. Generation of alveolar epithelial spheroids via isolated progenitor cells from human pluripotent stem cells. Stem Cell Rep. 2014;3:394-403.

9. Huang SXL, Islam MN, O’Neill J, Hu Z, Yang YG, Chen YW, et al. Efficient generation of lung and airway epithelial cells from human pluripotent stem cells. Nat Biotechnol. 2014;32:84-91.

10. Firth AL, Dargitz CT, Qualls SJ, Menon T, Wright R, Singer O, et al. Generation of multiciliated cells in functional airway epithelia from human induced pluripotent stem cells. Proc Natl Acad Sci USA. 2014;20:111.

11. Konishi S, Gotoh S, Tateishi K, Yamamoto Y, Korogi Y, Nagasaki $\mathrm{T}$, et al. Directed induction of functional multi-ciliated cells in proximal airway epithelial spheroids from human pluripotent stem cells. Stem Cell Rep. 2016;6:18-25.

12. Langer R, Vacanti JP. Tissue engineering. Science. 1993;260:920-6.

13. Ott HC, Matthiesen TS, Goh SK, Black LD, Kren SM, Netoff TI, et al. Perfusion-decellularized matrix: using nature's platform to engineer a bioartificial heart. Nat Med. 2008;14:213-21.

14. Sender R, Fuchs S, Milo R. Revised estimates for the number of human and bacteria cells in the body. PLoS Biol. 2016;14:1-14.

15. Ingber DE. Mechanical control of tissue morphogenesis during embryological development. Int J Dev Biol. 2006;50:255-66.

16. Rozario T, DeSimone DW. The extracellular matrix in development and morphogenesis: a dynamic view. Dev Biol. 2010;341:126-40.

17. DeQuach JA, Mezzano V, Miglani A, Lange S, Keller GM, Sheikh F, et al. Simple and high yielding method for preparing tissue specific extracellular matrix coatings for cell culture. PLoS ONE. 2010;5:1-11.

18. Clark RAF, Mason RJ, Folkvord JM, McDonald JA. Fibronectin mediates adherence of rat alveolar type II epithelial cells via the fibroblastic cell-attachment domain. J Clin Invest. 1986;77:1831-40.

19. Vorotnikova E, McIntosh D, Dewilde A, Zhang J, Reing JE, Zhang L, et al. Extracellular matrix-derived products modulate endothelial and progenitor cell migration and proliferation in vitro and stimulate regenerative healing in vivo. Matrix Biol. 2010;29:690-700.

20. Ohta R, Niwa A, Taniguchi Y, Suzuki NM, Toga J, Yagi E, et al. Laminin-guided highly efficient endothelial commitment from human pluripotent stem cells. Sci Rep. 2016;6:1-12.

21. Sougawa N, Miyagawa S, Fukushima S, Yokoyama J, Kitahara M, Harada A, et al. Laminin-511 supplementation enhances stem cell localization with suppression in the decline of cardiac function in acute infarct rats. Transplantation. 2019;103:e119-e12727.

22. Järveläinen H, Sainio A, Koulu M, Wight TN, Penttinen R. Extracellular matrix molecules: potential targets in pharmacotherapy. Pharmacol Rev. 2009;61:198-223.

23. Miyazaki T, Futaki S, Suemori H, Taniguchi Y, Yamada M, Kawasaki M, et al. Laminin E8 fragments support efficient adhesion and expansion of dissociated human pluripotent stem cells. Nat Commun. 2012;3:1210-36.

24. Petersen TH, Calle EA, Zhao L, Lee EJ, Gui L, Raredon MB, et al. Tissue-engineered lungs for in vivo implantation. Science. 2010;329:538-41.

25. Ott HC, Clippinger B, Conrad C, Schuetz C, Pomerantseva I, Ikonomou L, et al. Regeneration and orthotopic transplantation of a bioartificial lung. Nat Med. 2010;16:927-33.

26. Uriarte JJ, Uhl FE, Rolandsson Enes SE, Pouliot RA, Weiss DJ. Lung bioengineering: advances and challenges in lung decellularization and recellularization. Curr Opin Organ Transplant. 2018;23:673-8.

27. Wallis JM, Borg ZD, Daly AB, Deng B, Ballif BA, Allen GB, et al. Comparative assessment of detergent-based protocols for mouse lung de-cellularization and re-cellularization. Tissue Eng Part C Methods. 2012;18:420-32.

28. Nagao RJ, Ouyang Y, Keller R, Lee C, Suggs LJ, Schmidt CE. Preservation of capillary-beds in rat lung tissue using optimized chemical decellularization. J Mater Chem B. 2013;1:4801-8.

29. Tsuchiya T, Balestrini JL, Mendez J, Calle EA, Zhao L, Niklason LE. Influence of $\mathrm{pH}$ on extracellular matrix preservation during lung decellularization. Tissue Eng Part C Methods. 2014;20:1028-36.

30. Nichols JE, La Francesca S, Niles JA, Vega SP, Argueta LB, Frank L, et al. Production and transplantation of bioengineered lung into a large-animal model. Sci Transl Med. 2018;20:10.

31. Tsuchiya T, Mendez J, Calle EA, Hatachi G, Doi R, Zhao L, et al. Ventilation-based decellularization system of the lung. Biores Open Access. 2016;5:118-26.

32. Dorrello NV, Guenthart BA, O'Neill JD, Kim J, Cunningham $\mathrm{K}$, Chen Y-W, et al. Functional vascularized lung grafts for lung bioengineering. Sci Adv. 2017;3:e1700521.

33. Guyette JP, Charest JM, Mills RW, Jank BJ, Moser PT, Gilpin $\mathrm{SE}$, et al. Bioengineering human myocardium on native extracellular matrix. Circ Res. 2016;118:56-72.

34. Mazza G, Rombouts K, Rennie Hall A, Urbani L, Vinh Luong T, Al-Akkad W, et al. Decellularized human liver as a natural 3D-scaffold for liver bioengineering and transplantation. Sci Rep. 2015;5:1-15.

35. Song JJ, Guyette JP, Gilpin SE, Gonzalez G, Vacanti JP, Ott HC. Regeneration and experimental orthotopic transplantation of a bioengineered kidney. Nat Med. 2013;19:646-51.

36. Goh SK, Bertera S, Olsen P, Candiello JE, Halfter W, Uechi G, et al. Perfusion-decellularized pancreas as a natural 3D scaffold for pancreatic tissue and whole organ engineering. Biomaterials. 2013;34:6760-72.

37. Kitano K, Schwartz DM, Zhou H, Gilpin SE, Wojtkiewicz $\mathrm{GR}$, Ren X, et al. Bioengineering of functional human induced pluripotent stem cell-derived intestinal grafts. Nat Commun. 2017;20:8.

38. Gerli MFM, Guyette JP, Evangelista-Leite D, Ghoshhajra BB, Ott HC. Perfusion decellularization of a human limb: a novel platform for composite tissue engineering and reconstructive surgery. PLoS ONE. 2018;13:1-18.

39. Zhou H, Kitano K, Ren X, Rajab TK, Wu M, Gilpin SE, et al. Bioengineering human lung grafts on porcine matrix. Ann Surg. 2018;267:590-8.

40. Kawase I, Ozaki S, Yamashita H, Uchida S, Nozawa Y, Matsuyama T, et al. Aortic valve reconstruction of unicuspid aortic valve 
by tricuspidization using autologous pericardium. Ann Thorac Surg. 2012;94:1180-4.

41. Chen G, Lv Y. Matrix elasticity-modified scaffold loaded with SDF- $1 \alpha$ improves the in situ regeneration of segmental bone defect in rabbit radius. Sci Rep. 2017;7:1-12.

42. Gilpin SE, Li Q, Evangelista-Leite D, Ren X, Reinhardt DP, Frey $\mathrm{BL}$, et al. Fibrillin-2 and Tenascin-C bridge the age gap in lung epithelial regeneration. Biomaterials. 2017;140:212-9.

43. Gilpin SE, Guyette JP, Gonzalez G, Ren X, Asara JM, Mathisen DJ, et al. Perfusion decellularization of human and porcine lungs: Bringing the matrix to clinical scale. J Hear Lung Transplant. 2014;33:298-308.

44. Ren X, Moser PT, Gilpin SE, Okamoto T, Wu T, Tapias LF, et al. Engineering pulmonary vasculature in decellularized rat and human lungs. Nat Biotechnol. 2015;33:1097-102.

45. Wagner DE, Bonenfant NR, Parsons CS, Sokocevic D, Brooks EM, Borg ZD, et al. Comparative decellularization and recellularization of normal versus emphysematous human lungs. Biomaterials. 2014;35:3281-97.

46. Cornwell KG, Landsman A, James KS. Extracellular matrix biomaterials for soft tissue repair. Clin Podiatr Med Surg. 2009;26:507-23.

47. Daly KA, Stewart-Akers AM, Hara H, Ezzelarab M, Long C, Cordero K, et al. Effect of the ogal epitope on the response to small intestinal submucosa extracellular matrix in a nonhuman primate model. Tissue Eng Part A. 2009;15:3877-88.

48. Stahl EC, Bonvillain RW, Skillen CD, Burger BL, Hara H, Lee W, et al. Evaluation of the host immune response to decellularized lung scaffolds derived from $\alpha$-Gal knockout pigs in a non-human primate model. Biomaterials. 2018;187:93-104.

49. Scarritt ME, Pashos NC, Motherwell JM, Eagle ZR, Burkett BJ, Gregory AN, et al. Re-endothelialization of rat lung scaffolds through passive, gravity-driven seeding of segment-specific pulmonary endothelial cells. J Tissue Eng Regen Med. 2018;12:e786-806.

50. Weber DJ, Wilkes DS. The role of autoimmunity in obliterative bronchiolitis after lung transplantation. Am J Physiol Cell Mol Physiol. 2012;304:L307-L311311.

51. Gilpin SE, Charest JM, Ren X, Tapias LF, Wu T, EvangelistaLeite D, et al. Regenerative potential of human airway stem cells in lung epithelial engineering. Biomaterials. 2016;108:111-9.

52. Gilpin SE, Ren X, Okamoto T, Guyette JP, Mou H, Rajagopal J, et al. Enhanced lung epithelial specification of human induced pluripotent stem cells on decellularized lung matrix. Ann Thorac Surg. 2014;98:1721-9.

53. Ghaedi M, Le AV, Hatachi G, Beloiartsev A, Rocco K, Sivarapatna A, et al. Bioengineered lungs generated from human iPSCsderived epithelial cells on native extracellular matrix. J Tissue Eng Regen Med. 2018;12:e1623-e16351635.

54. Kim J, Guenthart B, O'Neill JD, Dorrello NV, Bacchetta M, Vunjak-Novakovic G. Controlled delivery and minimally invasive imaging of stem cells in the lung. Sci Rep. 2017;7:1-13.

55. Doi R, Tsuchiya T, Mitsutake N, Nishimura S, Matsuu-Matsuyama M, Nakazawa Y, et al. Transplantation of bioengineered rat lungs recellularized with endothelial and adipose-derived stromal cells. Sci Rep. 2017;7:1-15.
56. Charest JM, Okamoto T, Kitano K, Yasuda A, Gilpin SE, Mathisen DJ, et al. Design and validation of a clinical-scale bioreactor for long-term isolated lung culture. Biomaterials. 2015;52:79-877.

57. Raredon MSB, Rocco KA, Gheorghe CP, Sivarapatna A, Ghaedi $\mathrm{M}$, Balestrini JL, et al. Biomimetic culture reactor for whole-lung. Engineering. 2016;5:72-83.

58. Linacre V, Cypel M, Machuca T, Nakajima D, Hashimoto K, Zamel R, et al. Importance of left atrial pressure during ex vivo lung perfusion. J Hear Lung Transplant. 2016;35:808-14.

59. Sanchez-Esteban J, Cicchiello LA, Wang Y, Tsai SW, Williams LK, Torday JS, et al. Mechanical stretch promotes alveolar epithelial type II cell differentiation. J Appl Physiol. 2001;91:589-95.

60. Petersen TH, Calle EA, Colehour MB, Niklason LE. Bioreactor for the long-term culture of lung tissue. Cell Transplant. 2011;20:1117-26.

61. Ren X, Tapias LF, Jank BJ, Mathisen DJ, Lanuti M, Ott HC. Exvivo non-invasive assessment of cell viability and proliferation in bio-engineered whole organ constructs. Biomaterials. 2015;52:103-12.

62. Dellatore SM, Garcia AS, Miller WM. Mimicking stem cell niches to increase stem cell expansion. Curr Opin Biotechnol. 2008;19:534-40.

63. Dye BR, Dedhia PH, Miller AJ, Nagy MS, White ES, Shea LD, et al. A bioengineered niche promotes in vivo engraftment and maturation of pluripotent stem cell derived human lung organoids. Elife. 2016;5:1-18.

64. Xu H, Wang B, Ono M, Kagita A, Fujii K, Sasakawa N, et al. Targeted disruption of HLA genes via CRISPR-Cas9 generates iPSCs with enhanced immune compatibility. Cell Stem Cell. 2019;24(566-578):e7.

65. Song JJ, Kim SS, Liu Z, Madsen JC, Mathisen DJ, Vacanti JP, et al. Enhanced in vivo function of bioartificial lungs in rats. Ann Thorac Surg. 2011;92:996-8.

66. Mishra DK, Thrall MJ, Baird BN, Ott HC, Blackmon SH, Kurie JM, et al. Human lung cancer cells grown on acellular rat lung matrix create perfusable tumor nodules. Ann Thorac Surg. 2012;93:1075-81.

67. Vishnoi M, Mishra DK, Thrall MJ, Kurie JM, Kim MP. Circulating tumor cells from a 4-dimensional lung cancer model are resistant to cisplatin. J Thorac Cardiovasc Surg. 2014;148:1056-64.

68. Mishra DK, Creighton CJ, Zhang Y, Gibbons DL, Kurie JM, Kim MP. Gene expression profile of A549 cells from tissue of $4 \mathrm{D}$ model predicts poor prognosis in lung cancer patients. Int $\mathrm{J}$ Cancer. 2014;134:789-98.

69. Tapias LF, Gilpin SE, Ren X, Wei L, Fuchs BC, Tanabe KK, et al. Assessment of proliferation and cytotoxicity in a biomimetic three-dimensional model of lung cancer. Ann Thorac Surg. 2015;100:414-21.

70. Lin Y-M, Zhang A, Rippon HJ, Bismarck A, Bishop AE. Tissue engineering of lung: the effect of extracellular matrix on the differentiation of embryonic stem cells to pneumocytes. Tissue Eng Part A. 2009;16:1515-26.

Publisher's Note Springer Nature remains neutral with regard to jurisdictional claims in published maps and institutional affiliations. 